\title{
Sistema para Evaluar la Confiabilidad de Equipos Críticos en el Sector Industrial
}

\author{
Maira C. Gasca( ${ }^{(1)}$, Luis L. Camargo ${ }^{(2)}$ y Byron Medina(3) \\ (1) Universidad Antonio Nariño, Research in Energy and Materials, REM. Avenida Libertador Calle 14 No. \\ 13-55, Santa Marta, Colombia. (e-mail: magasca@uan.edu.co). \\ (2) Universidad del Magdalena, Grupo de Investigación en Desarrollo Electrónico y Aplicaciones Móviles, \\ GIDEAM. Carrera 32 No. 22-08, Santa Marta, Colombia. (e-mail: Icamargoa@unimagdalena.edu.co). \\ (3) Universidad Francisco de Paula Santander, Grupo de Investigación y Desarrollo en \\ Telecomunicaciones, GIDT. Avenida Gran Colombia No. 12E-96, San José de Cúcuta, Colombia. \\ (e-mail: byronmedina@ufps.edu.co).
}

Recibido Nov. 29, 2016; Aceptado Ene. 27, 2017; Versión final Mar. 16, 2017, Publicado Ago. 2017

\begin{abstract}
Resumen
Se propone una herramienta para evaluar la confiabilidad de equipos críticos, con el objetivo de brindar criterios para mejorar la toma de decisiones en el mantenimiento. La herramienta primero jerarquiza los activos a través de un análisis de criticidad, identificando los equipos en función de los siguiente factores: i) su operación y producción; ii) frecuencia, costo y tiempo de reparación de las fallas; y iii) el impacto en la seguridad y salud del operador. A los equipos críticos se les construye un catálogo de fallas, mediante el uso de la metodología de análisis del modo y efecto de la falla, creando un registro confiable. Con estos datos se analiza y modela la confiabilidad de los equipos utilizando las distribuciones de Weibull, Log Normal y Normal, para determinar el tiempo medio entre fallas. La herramienta se probó en una industria transformadora de plástico, dando como resultados la identificación de la extrusora como el equipo más crítico. También se confecciono el catálogo de fallas de varios equipos, y se calcula el tiempo medio entre fallas de forma automática y dinámica, a partir del historial de las fallas. Este procedimiento ofrece indicadores para tomar decisiones que eviten paradas no planificadas de los equipos.
\end{abstract}

Palabras clave: mantenimiento, criticidad, disponibilidad, falla, Weibull

\section{System to Assess the Reliability of Critical Equipment in the Industrial Sector}

\begin{abstract}
A tool is proposed to evaluate the reliability of critical equipment, to provide criteria to improve decision making in maintenance. First, the tool hierarchizes the pieces of equipment through a criticality analysis, identifying the equipment in function of the following factors: i) its operation and production; ii) frequency, cost, repair time of the failures; and iii) the impact on the safety and health of the operator. A catalog of faults is built for each of the pieces of critical equipment, using the methodology of analysis of the mode and effect of the fault, creating a reliable record. With these data, the reliability of the equipment is analyzed and it is modeled using the Weibull, Normal and Log Normal distributions to determine the average time between faults. The tool was tested in a plastic processing industry, resulting in the identification of the extruder as the most critical equipment. In addition, a catalog of failures of various pieces of equipment was done, and calculation of the mean time between failures in automatic and dynamic form using the historical record of failures was done. This procedure offers indicators for decision-making, avoiding unplanned stops of the equipment.
\end{abstract}

Keywords: maintenance, criticality, reliability, failure, Weibull 


\section{INTRODUCCIÓN}

Los procesos asociados a la gestión del mantenimiento deben ser innovadores, metódicos, planeados y liderados por la alta administración, involucrando desde el personal operativo hasta los gerentes (Espinosa et al., 2008); sin embargo, en muchas ocasiones la administración no se preocupa por realizar una planificación adecuada del mantenimiento, incumpliendo los estándares de desempeño de sus procesos, debido a la baja confiabilidad en los activos de producción, repercutiendo en los costos globales de operación, en la vida útil de la maquinaria y en el nivel competitivo de la industria (Espinosa et al., 2012; Chemweno et al., 2015). La gestión del mantenimiento debe ser eficaz, eficiente y oportuno, y debe alinearse con los objetivos y las necesidades de la empresa, minimizando los costos indirectos y directos asociados; además, la falta de planificación impide ejecutar las actividades de mantenimiento efectivas de forma proactiva (Viveros et al., 2013; Pintelon y Gelders, 1992). Al desarrollar solo mantenimientos por averías, los costos asociados a las fallas se aumentan, las paradas no planificadas impactan los costos de producción no solo por la interrupción del proceso, sino también por el desperdicio de material, defectos de calidad en los productos fabricados, riesgos de accidente por condiciones inseguras, y otros factores asociados a la baja confiabilidad de los activos (Duffuaa et al., 2009; Arata, 2009). Las organizaciones competitivas buscan altos niveles de confiabilidad operacional en sus procesos, productos y servicios, para aumentar la seguridad y efectividad de sus sistemas (Zio, 2009; Flores et al., 2010).

La confiabilidad operacional, algunos autores la definen como la capacidad de una organización para cumplir sus funciones de manera óptima, durante un período de tiempo, bajo un contexto operacional específico (Holmberg et al., 1991; Altuger y Chassapis, 2009; Puthillath y Sasikumar, 2012; Bazovsky, 2004; Mora, 2009). La confiabilidad operacional está determinada por la confiabilidad del equipo, la confiabilidad humana, la confiabilidad del proceso y la mantenibilidad (Altmann, 2009). La medida de la confiabilidad de un equipo es la frecuencia con la cual ocurren las fallas en el tiempo; si no hay fallas, el equipo es totalmente confiable; si la frecuencia de fallas es muy baja, la confiabilidad del equipo es aun aceptable; pero si la frecuencia de fallas es muy alta, el equipo es poco confiable (Bazovsky, 2004). La confiabilidad de un equipo se puede estimar con el análisis probabilístico del tiempo para la falla o el historial de fallas (Haldar y Mahadevan, 2000), o con el análisis probabilístico del proceso de deterioro o física de la falla (Cruse, 1997). Ambos métodos caracterizan la probabilidad de la falla para pronosticar y establecer acciones proactivas que eviten o mitiguen su efecto (Sun et al., 2012; Yañez et al., 2004; Yañez et al., 2012). Este trabajo, estima la confiabilidad a partir del historial de las fallas.

Para estimar la confiabilidad a partir del historial de las fallas, es indispensable que éstas estén codificadas y registradas en un formato único, con todos los atributos requeridos (Lowe y Lewis, 1983; Campos et al., 2014). Por esto se propone la construcción del catálogo de fallas, elaborados con base en el Análisis del Modo y Efecto de la Falla, AMEF, para identificar metódicamente la función del activo y del componente que falla, las fallas asociadas, el impacto del desempeño global y la causa de la falla. La elaboración de estos catálogos requiere del conocimiento sobre la construcción y la operación del equipo, resultando en un procedimiento costoso para las pequeñas y medianas industrias (Price et al., 1995); por tal motivo, este artículo sugiere solo analizar equipos críticos, haciendo relevante definir previamente los niveles de criticidad de los equipos, para enfocar los esfuerzos y recursos hacia las áreas en donde sea más importante y/o necesario. La determinación del nivel de criticidad se puede establecer a partir de un análisis de: la frecuencia por consecuencia, la consecuencia versus la complejidad, o el impacto global; estos tipos de análisis evalúan criterios como seguridad, ambiente, costos de operación y mantenimiento, tiempo de reparación, continuidad operativa, calidad, frecuencia de las fallas, entre otros, obteniendo expresiones matemáticas específica para cada organización dependiendo de sus objetivos (Solomon y Oldach, 2016; Castillo-Serpa et al., 2009). En este trabajo, se emplea el método de frecuencia por consecuencia, y se personalizan los criterios de la consecuencia.

El momento de la falla de un equipo no puede determinarse con certeza, sin embargo, existen en la literatura científica varios trabajos que utilizan el comportamiento histórico de las fallas y la probabilidad y estadística, para estimar la probabilidad de ocurrencia del evento, disminuyendo así la incertidumbre asociada a las labores de prevención de averías y optimizando la gestión de mantenimiento (Canfield, 1986; Ge et al., 2013; Duan et al., 2015); también existen diversas publicaciones sobre el análisis de criticidad, y el AMEF, (Price et al., 1995; Sutrisno et al., 2016; Valdes, 2012; Jiang et al., 2015), como herramientas para mejorar las acciones de mantenimiento. Este trabajo se diferencia de los mencionados, porque incorpora en una sola propuesta, tres herramientas del mantenimiento centrado en confiabilidad, como el análisis de criticidad, el AMEF, y el modelado de la confiabilidad; con el objetivo de crear un sistema que permita evaluar la confiabilidad de los equipos críticos en la industria, aportando criterios para mejorar la toma de decisiones en el mantenimiento, acorde a los patrones de falla del equipo y a su importancia en el contexto. El análisis de criticidad formula una ecuación matemática que asocia la frecuencia y la consecuencia, y que se aplica a través de un instrumento que pondera los criterios de la consecuencia de la falla de los equipos en una línea de producción 
industrial, para identificar los equipos a los que se les va a construir el catálogo de fallas. El instrumento para crear el AMEF se realiza con base en las normas ISO 14224, SAE JA1011 y SAE JA1012, y tiene el propósito de identificar los subsistemas y las partes del equipo, asociándoles la falla funcional y, la causa y el efecto de la falla, para generar el catálogo de las fallas de manera sistematizada. El modelado de la confiabilidad, se realiza a partir del análisis del historial de las fallas, identificando la distribución que más se ajusta, Weibull, Log Normal y Normal, para estimar el tiempo medio entre las fallas.

Este sistema se implementó de forma ágil, fácil y económica, utilizando una aplicación de hojas de cálculo, y se probó en una industria transformadora de plástico, obteniendo como resultado: la jerarquización de los equipos, los catálogos de fallas sistematizados de los equipos con criticidad alta, el Tiempo Medio Entre Fallas, MTBF, de los equipos o subsistemas del equipo, y la gráfica de la confiabilidad del equipo o subsistema. EI MTBF se calculó de forma dinámica a partir de los registros de las fallas y las acciones de mantenimiento preventivo y correctivo asociadas a éstas y no en la distribución uniforme de la vida útil del equipo. Resultados que son considerables en el rediseño de las labores de mantenimiento en esta industria.

\section{MÉTODOS}

El desarrollo del sistema parte de identificar los equipos que deben ser atendidos en función de su impacto sobre la operación; posteriormente a estos equipos se les construye un catálogo de fallas, creando una base de datos con información real referente a sus fallas; finalmente, con estos datos se procede al análisis y modelado de la confiabilidad.

\section{Identificación de los equipos críticos}

Para identificar los equipos críticos se realiza el análisis de criticidad, evaluando la frecuencia de la falla por su consecuencia, a través de la encuesta a un conjunto de personas previamente seleccionadas del área de mantenimiento y de operación, de la industria; la validación del instrumento se obtuvo a través del juicio de tres expertos en el área, y la confiabilidad por el método de Alfa Cronbach, con un coeficiente de 0,81. Con base en los resultados de las encuestas, se jerarquizan los equipos de la planta de producción, mediante la cuantificación de un índice de criticidad IC, a partir de las ecuaciones (1) y (2), y de la ponderación de las respuestas, según las tablas 1, 2, 3, 4, 5 y 6 . El IC, según la ecuación (1), es el producto de la frecuencia de fallas FF y la consecuencia de la falla CF, esta ecuación es ampliamente utilizada por la literatura.

$I C=F F \times C F$

La FF se evalúa a partir de la tasa estimada de incidencia de fallas. La ponderación de FF se muestra en la tabla 1.

Tabla 1: Ponderación del FF

\begin{tabular}{|c|c|}
\hline Frecuencia de Fallas (FF) & Valor \\
\hline \multicolumn{2}{|l|}{ ¿Qué tan frecuente son las fallas ocurridas? } \\
\hline Menos de 1 por año & 1 \\
\hline Entre 2 y 12 por año & 2 \\
\hline Entre 13 y 52 por año & 3 \\
\hline Más de 52 por año (más de una por semana) & 4 \\
\hline
\end{tabular}

La CF se evalúa a partir de la suma de: el impacto en la producción IP, el impacto en la seguridad y salud SS, los costos de reparación CR, el tiempo de reparación TR, y el tiempo de operación TO. Estos criterios fueron elegidos por los expertos que validaron el instrumento y las tablas de ponderación, considerando que son los más significativos en la evaluación de la consecuencia que puede ocasionar una falla en un equipo industrial. Finalmente, IC se calcula utilizando la ecuación (2).

$I C=F F \times(I P+S S+C R+T R+T O)$

EI IP registra porcentualmente la producción aproximada que se deja de obtener por día, debido a fallas ocurridas; el IP define la consecuencia inmediata de la ocurrencia de la falla, que puede representar un paro total o parcial de los quipos del sistema estudiado y al mismo tiempo el paro del proceso productivo de la unidad. La ponderación del IP se muestra en la tabla 2.

EI SS registra la ocurrencia de eventos en los cuales alguna persona puede resultar lesionada, para este indicador se sugiere obtener la información y colaboración del personal de: recursos humanos, comités 
paritarios y salud ocupacional de la empresa. La ponderación de SS se muestra en la tabla 3. El CR hace referencia al costo promedio por falla requerido para restituir el equipo a condiciones óptimas de funcionamiento, incluye labor, materiales y transporte. La ponderación del CR se muestra en la tabla 4.

Tabla 2: Ponderación del IP

\begin{tabular}{|l|c|}
\hline \multicolumn{1}{|c|}{ Impacto en la Producción (IP) } & Valor \\
\hline ¿Cuál es el impacto en la producción? & 1 \\
\hline Menor al $25 \%$ & 2 \\
\hline $25 \%$ de impacto & 3 \\
\hline $50 \%$ de impacto & 4 \\
\hline $75 \%$ de impacto
\end{tabular}

Tabla 3: Ponderación del SS

\begin{tabular}{|l|c|}
\hline \multicolumn{1}{|c|}{ Seguridad y Salud (SS) } & \multicolumn{2}{c|}{ Valor } \\
\hline \multicolumn{2}{|c|}{ ¿Cuál es la lesión más significativa que puede presentarse en la operación del equipo? } \\
\hline No hay algún riesgo de lesión & 1 \\
\hline Lesiones leves (son asistidas dentro de la fábrica, no hay incapacidad) & 2 \\
\hline Lesiones significativas (incapacidad entre 1 y 30 días) & 3 \\
\hline Lesiones de incapacidad parcial o permanente (mayor de 30 días) & 4 \\
\hline
\end{tabular}

Tabla 4: Ponderación del CR

\begin{tabular}{|c|c|}
\hline Costos de Reparación (CR) & Valor \\
\hline \multicolumn{2}{|c|}{ ¿Cuál es el costo anual del mantenimiento del equipo? } \\
\hline Menos de 1.000 dólares & 1 \\
\hline Entre 1.000 y 5.000 dólares & 2 \\
\hline Entre 5.001 y 10.000 dólares & 3 \\
\hline Más de 10.000 dólares & 4 \\
\hline
\end{tabular}

EI TR es el tiempo promedio por día empleado para reparar la falla, se considera desde que el equipo pierde su función hasta que esté disponible para cumplirla nuevamente. EI TR, mide la efectividad para restituir la unidad o unidades del sistema en estudio a condiciones óptimas de operatividad. La ponderación del TR se muestra en la tabla 5 .

Tabla 5: Ponderación del TR

\begin{tabular}{|c|c|}
\hline Tiempo de Reparación (TR) & Valor \\
\hline \multicolumn{2}{|c|}{ ¿Cuál es el tiempo promedio para reparar el equipo? } \\
\hline Menor a 4 horas & 1 \\
\hline Igual o mayor a 4 horas y menor a 6 horas & 2 \\
\hline Igual o mayor a 6 horas y menor a 12 horas & 3 \\
\hline Mayor a 12 horas & 4 \\
\hline
\end{tabular}

EI TO es el tiempo que dura el equipo en producción. La ponderación del TO se muestra en la tabla 6. Obtenido el valor del índice de criticidad de cada equipo, éstos se clasificaron como de alta criticidad entre (51-80), media criticidad (26-50) o baja criticidad (5-25), de acuerdo con la matriz de criticidad de la figura 1.

Tabla 6: Ponderación del TO

\begin{tabular}{|c|c|}
\hline Tiempo de Operación (TO) & Valor \\
\hline \multicolumn{2}{|l|}{ ¿Cuál es el tiempo de trabajo de esta máquina? } \\
\hline Opcionalmente & 1 \\
\hline Un turno de trabajo & 2 \\
\hline Dos turnos de trabajo & 3 \\
\hline Totalmente & 4 \\
\hline
\end{tabular}




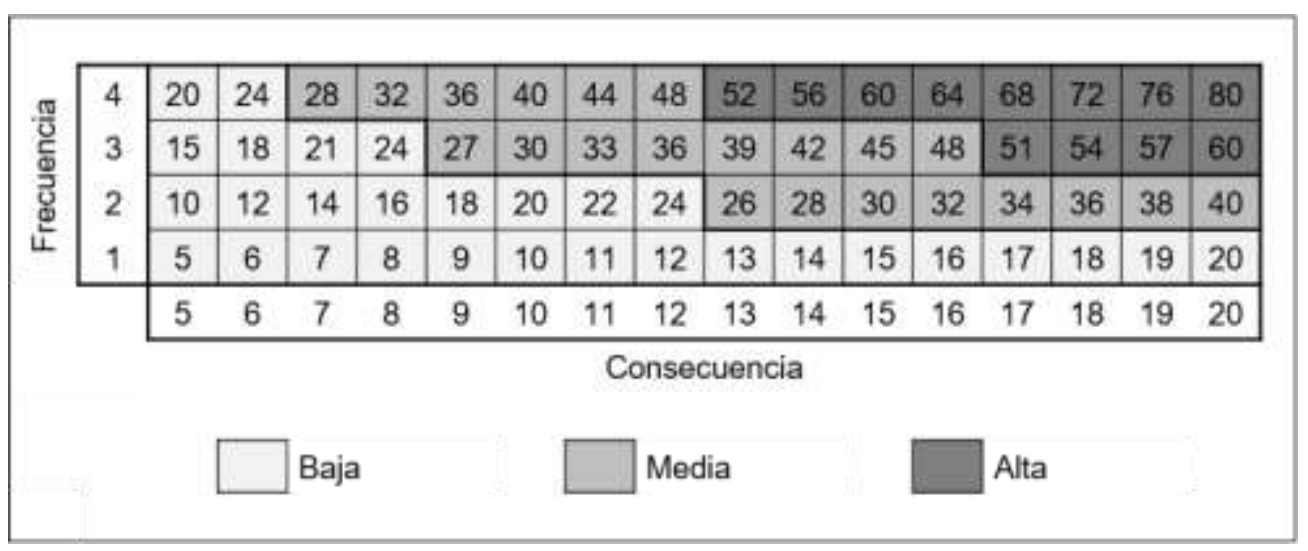

Fig. 1: Matriz de criticidad

\section{Catálogo de fallas}

Determinados los equipos críticos se realiza el catálogo de fallas para cada uno, relacionando ordenadamente las posibles fallas del equipo, con el objetivo de que las fallas presentadas sean registradas y descritas de la misma manera y, con todos los requerimientos para el análisis y modelado de la confiabilidad del mismo. Este catalogó se basa en una de las herramientas del Mantenimiento, como lo es el Análisis de Modo y Efecto de la Falla AMEF; identificando metódicamente la función del activo y del componente que falla, las fallas asociadas, el impacto en el desempeño global y la causa de la falla. El instrumento diseñado para realizar el AMEF se muestra en la tabla 7. La elaboración e implementación de este instrumento se basa en las normas: ISO 14224, SAE JA1011 y SAE JA1012.

Tabla 7: Instrumento para el AMEF

\begin{tabular}{|c|c|c|c|c|c|c|c|}
\hline \multicolumn{10}{|c|}{ AMEF del equipo } \\
\hline Subsistema & $\begin{array}{c}\text { Función del } \\
\text { activo del } \\
\text { subsistema }\end{array}$ & $\begin{array}{c}\text { Falla } \\
\text { funcional }\end{array}$ & $\begin{array}{c}\text { Modos } \\
\text { de falla }\end{array}$ & $\begin{array}{c}\text { Parte de } \\
\text { equipo } \\
\text { afectada }\end{array}$ & $\begin{array}{c}\text { Causa } \\
\text { de } \\
\text { falla }\end{array}$ & $\begin{array}{c}\text { Efecto de falla } \\
\text { y } \\
\text { consecuencia }\end{array}$ & $\begin{array}{c}\text { Actividad de } \\
\text { mantenimiento } \\
\text { recomendada }\end{array}$ \\
\hline
\end{tabular}

De la ISO 14224 se hereda el concepto que si el equipo es de alta complejidad, éste se divide en sistema, subsistema, ítem mantenible y componente de detalle, según el grado de complejidad (ISO, 2006). Basados en SAE JA1012 se define el activo, esto incluye la selección del activo/sistema, la definición de sus límites, y la identificación del nivel de detalle más apropiado, al cual se llevará a cabo el análisis (SAE, 2011). Definido el activo y, conforme a la SAE JA1011, se identifican las funciones deseadas y los estándares de desempeño asociados al activo en su contexto operacional presente (funciones). Posteriormente, de acuerdo con la SAE JA1011, se define la función de la falla determinando los estados de falla asociados con cada función; y se definen los modos de falla teniendo en cuenta la sección 5.3 de la norma: identificar los modos de falla, establecer que se entiende por probable, niveles de causalidad, fuentes de información, y tipos de modos de falla (SAE, 2009). Terminado el AMEF se realiza el catálogo de fallas, permitiendo al operario registrar las fallas de forma sistematizada, seleccionando cada opción disponible (Equipos, Subsistema, Falla funcional, Modo de falla, Parte equipo y Causa falla), según el AMEF del equipo que presentó la falla. Las fallas son registradas en el sistema de información como se muestra en la tabla 8.

Tabla 8: Fallas registradas utilizando el catálogo

\begin{tabular}{|c|c|c|c|c|c|c|c|c|}
\hline \multicolumn{10}{|c|}{ Fallas registradas según catálogo } \\
\hline$\#$ & $\begin{array}{c}\text { Fecha } \\
\text { falla }\end{array}$ & $\begin{array}{c}\text { Horas } \\
\text { duración }\end{array}$ & Equipo & Subsistema & $\begin{array}{c}\text { Falla } \\
\text { funcional }\end{array}$ & $\begin{array}{c}\text { Modo } \\
\text { falla }\end{array}$ & $\begin{array}{c}\text { Parte } \\
\text { equipo }\end{array}$ & $\begin{array}{c}\text { Causa } \\
\text { falla }\end{array}$ \\
\hline
\end{tabular}

\section{Modelo para el manejo de la confiabilidad}

La medida de la confiabilidad de un equipo es la frecuencia con la cual ocurren las fallas en el tiempo. Para evaluar la confiabilidad se adaptó el modelo estadístico propuesto por Mora (Mora, 2009), utilizando las distribuciones paramétricas de Weibull, Log Normal y Normal. El modelo estima el tiempo medio entre fallas MTBF de un sistema reparable, el pronóstico de curvas de densidad de fallas y el análisis de los parámetros de la distribución elegida; en función de la distribución validada por el método de bondad de ajuste. Inicialmente se calcula el tiempo entre fallas, (Time Between Failures, TBF), con base en el historial de fallas registrado, usando el catálogo de fallas previamente descrito. EI TBF, según la ecuación (3), se calcula a partir 
del tiempo útil en que el equipo funciona correctamente, (Time Up, UT), y se desprecia el tiempo no operativo ( Time Down, DT) debido a que UT es mucho mayor a DT, en el contexto industrial. Posteriormente, se cuentan las fallas presentes y se organizan de forma ascendente, según el TBF.

$T B F \cong U T$

Se estima la no confiabilidad para cada falla a partir de la función de Weibull, utilizando el método de rango de medianas, el cual es un estimador no paramétrico basado en el orden de las fallas, este es el valor que la probabilidad de falla debe tener para la enésima falla, ecuación (4)

$F(t i)=\frac{\frac{i}{(n-i+1)}}{F_{1-\alpha, 2(2-i+1), 2 i}+\frac{i}{(n-i+1)}}$

Donde $F(t i)$ es el rango de mediana para un nivel de confianza (1- $\alpha)$, ti es el tiempo entre fallas TBF, $\alpha$ es el nivel de significancia y toma el valor de 0,5 para este estimador, $i$ es el orden de la falla, $n$ el número total de datos de la muestra; Fa, v1, v2 son valores críticos de la distribución $F$, evaluada en el nivel de significancia $\alpha$ y con grados de libertad $v 1$ y $v 2$.

Se transforman los valores de $\mathrm{Y}$ y $\mathrm{X}$ para el caso de no confiabilidad, según las ecuaciones (5) y (6), y se escoge el método de mínimos cuadrados para calcular los parámetros de la distribución.

$Y i=\operatorname{Ln}\left(\operatorname{Ln}\left(\frac{1}{1-F(t i)}\right)\right)$

$X i=\operatorname{Ln}(t i)$

Se mide la calidad de la alineación obtenida, utilizando el coeficiente de correlación $r$ y el coeficiente de determinación muestral $r^{2}$. Si la condiciones de: $0,95 \leq r \leq 1$ y $0,9 \leq r^{2} \leq 1$ se cumplen, los datos son válidos, y se calculan los parámetros de la distribución de Weibull. Gamma ( $\mathrm{v}$ ): parámetro de posición, indica el lapso en el cual la probabilidad de falla es nula, para el caso se asume que es cero. Beta ( $\beta$ ): parámetro de forma, refleja la dispersión de los datos y determina la forma que toma la distribución, y es igual a la pendiente de la recta estimada con la técnica de los mínimos cuadrados, $b$. Alfa $(\alpha)$ : parámetro de escala, o característica de vida útil, ver la ecuación (7).

$\alpha=e^{\frac{-a}{b}}$

Donde a es el intercepto con el eje Y según la recta estimada con la técnica de los mínimos cuadrados. La distribución de Weibull se representa mediante la ecuación (8).

$F(t)=1-e^{\left(-\int \lambda(t) d t\right)}=1-e^{\left(-\frac{t-t_{0}}{\alpha}\right)^{\beta}}$

Se asume to $=0$, para fallas registradas a partir del inicio del análisis. Calculados los parámetros $\alpha$ y $\beta$ se realiza la prueba de bondad de ajuste por el método de Kolmogorov-Smirnov, K-S; para determinar si los datos de fallas se ajustan a la distribución de Weibull. Si los datos se ajustan, se realiza el cálculo del Tiempo Medio Entre las Fallas, MTBF, de acuerdo con la ecuación (9). Donde 「 es la función Gamma para Weilbull.

$M T B F=\alpha \times \Gamma\left(1+\frac{1}{\beta}\right)$

Además, se realiza el análisis de confiabilidad del equipo y se grafican las curvas de confiabilidad, según la ecuación (10).

$R(t)=e^{-\left(\frac{t}{\alpha}\right)^{\beta}}$ 
Si los datos no cumplen la condición de $0,95 \leq r \leq 1$ y $0,9 \leq r^{2} \leq 1$, se evalúa el valor de $\beta$. Si $\beta$ se encuentra entre 0 y 1,95 se realiza el análisis con la distribución Log Normal. Si $\beta$ es mayor que 1,95 se realiza el análisis utilizando la distribución Normal. Para la Log Normal se transforman los valores de $\mathrm{Y}$ y $\mathrm{X}$ para no confiabilidad, siguiendo las ecuaciones (11) y (12).

$Y i=\Phi^{-1}[F(t i)]$

$X i=\operatorname{Ln}(t i)$

Donde $\Phi^{-1}$ es el inverso de la distribución normal estándar, $F(t i) ; X i$ es el estimado de la no confiablidad y ti es el tiempo entre falla. A los nuevos valores de Xi y Yi se aplica el método de mínimos cuadrados y, se calcula $\beta$ que es la pendiente y a que es el intercepto de la recta alineada. Se realiza la prueba de alineación, calculando el coeficiente de correlación $r$ y el coeficiente de determinación muestral $r^{2}$. Si la prueba es satisfactoria, se calculan los parámetros de la distribución Log Normal $\sigma$ y $\mu$, utilizando las ecuaciones (13) y (14).

$\sigma=\frac{1}{\beta}$

$\mu=-a \sigma$

Donde $\sigma$ es la desviación estándar. La distribución Log Normal se representa por la ecuación (15).

$F(t)=\frac{e^{\left[-\frac{(\ln (t)-\mu)^{2}}{2 \sigma^{2}}\right]}}{t \sigma \sqrt{2 \pi}}$

Se realiza la prueba de bondad de ajuste por el método de Kolmogorov-Smirnov. Si las pruebas de alineación y bondad se cumplen, entonces se calcula el MTBF según la distribución Log Normal, siguiendo la ecuación (16); y se grafica la curva de confiabilidad.

$M T B F=e^{\left(\mu+\frac{\sigma^{2}}{2}\right)}$

Si las pruebas de alineación y de bondad no se cumplen, entonces los datos no son válidos, y se debe utilizar la distribución Normal. Para la distribución Normal los valores de Y y X están dados por las ecuaciones (17) y (18).

$Y i=\Phi^{-1}[F(t i)]$

$X i=(t i)$

Se aplica nuevamente mínimos cuadrados y se obtienen los parámetros de la distribución utilizando las ecuaciones (13) y (14). La distribución Normal está dada por la ecuación (19), y el MTBF es el parámetro $\mu$.

$F(t)=\frac{e^{\left[-\frac{(t-\mu)^{2}}{2 \sigma^{2}}\right]}}{\sigma \sqrt{2 \pi}}$

Si las pruebas de alineación y bondad no se cumplen, entonces los datos no son válidos y no es posible calcular la confiabilidad y el MTBF.

\section{RESULTADOS}

El sistema se implementó de forma ágil, fácil y económica, utilizando una aplicación de hojas de cálculo, en una industria transformadora de plástico que tiene el diagrama de procesos que se muestra en la Figura 2. 


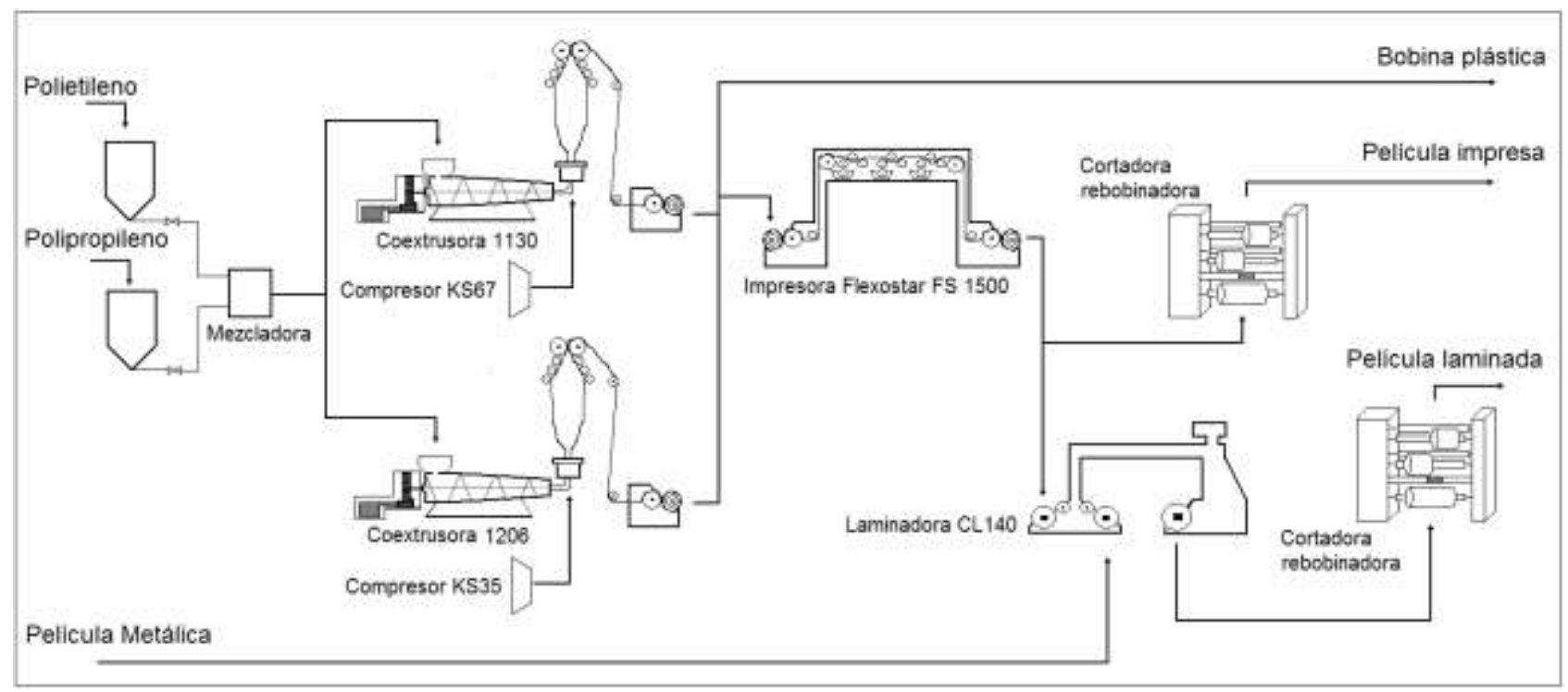

Fig. 2: Diagrama de flujo del proceso

\section{Clasificación de los equipos según el valor del IC}

Se identificaron los equipos de producción de la empresa, se calculó el índice de criticidad para cada equipo, y se procedió a clasificar los equipos como de alta, media o baja criticidad, como se muestra en la tabla 9.

Tabla 9: Equipos clasificados según su criticidad

\begin{tabular}{|c|c|c|c|c|c|}
\hline \multicolumn{6}{|c|}{ Equipos de la planta de producción } \\
\hline Ref. & Máquina & Descripción & Fabricante & $I C$ & Criticidad \\
\hline 1130 & Extrusora & Coextrusora 1130 & Ghioldi & 57 & \multirow{2}{*}{ Alta } \\
\hline 1206 & Extrusora & Coextrusora 1206 & Ghioldi & 54 & \\
\hline 1003 & Cortadora rebobinadora & Cortadora rebobinadora 332006 & Profama & 42 & \multirow{4}{*}{ Media } \\
\hline 2141 & Impresora & Impresora Flexostar FS 1500 & Comexi & 48 & \\
\hline 1400 & Cortadora & Cortadora Corex & Gianiflli L\&G & 36 & \\
\hline 1004 & Compresor & Compresor KS67 & Air Krone & 44 & \\
\hline 2052 & Laminadora & Laminadora CL140 & Comexi & 24 & \multirow{7}{*}{ Baja } \\
\hline 2144 & Cortadora rebobinadora & Cortadora rebobinadora KSC-120 & Comexi & 18 & \\
\hline 1005 & Compresor & Compresor KS35 & Bottarin & 24 & \\
\hline 7146 & Compactadora & Compacta material & Bottarin & 22 & \\
\hline 6140 & Mezcladora & Mezcla material & Chiller Alfaliq & 20 & \\
\hline 1002 & Montadora & Montadora Harley & Harley & 17 & \\
\hline 1001 & Estabilizador de voltaje & Regula el voltaje & Lef & 14 & \\
\hline
\end{tabular}

Determinado el grado de criticidad de los equipos de la empresa, se construyeron solo los catálogos de fallas de las extrusoras 1130 y 1206, enfocando los recursos de mantenimiento en los equipos críticos. Para efectos de la publicación, solo se relacionan los detalles de la construcción del catálogo de fallas de la extrusora 1130.

\section{Catálogo de fallas de la máquina extrusora Ghioldi 1130}

Como esta máquina es compleja (ver figura 3), se aplicó el concepto de la norma ISO 14224 y se dividió el quipo en subsistemas. Los subsistemas identificados en la extrusora Ghioldi 1130 son: Gravimétrico Doteco, Co-extrusor modelo 40A, 40B y 50C, Sistema IBC Inyectora de Burbuja Controlada, Halador Oscilante TKF 1200, Bobinador automático, y Tratador electrónico. La tabla 10 describe el subsistema Co-extrusor modelo 40A, 40B y 50C, para ejemplificar cómo se deben enunciar las partes del subsistema y los ítems mantenibles o reemplazables de cada parte; la descripción de los demás subsistemas siguió el mismo formato. 

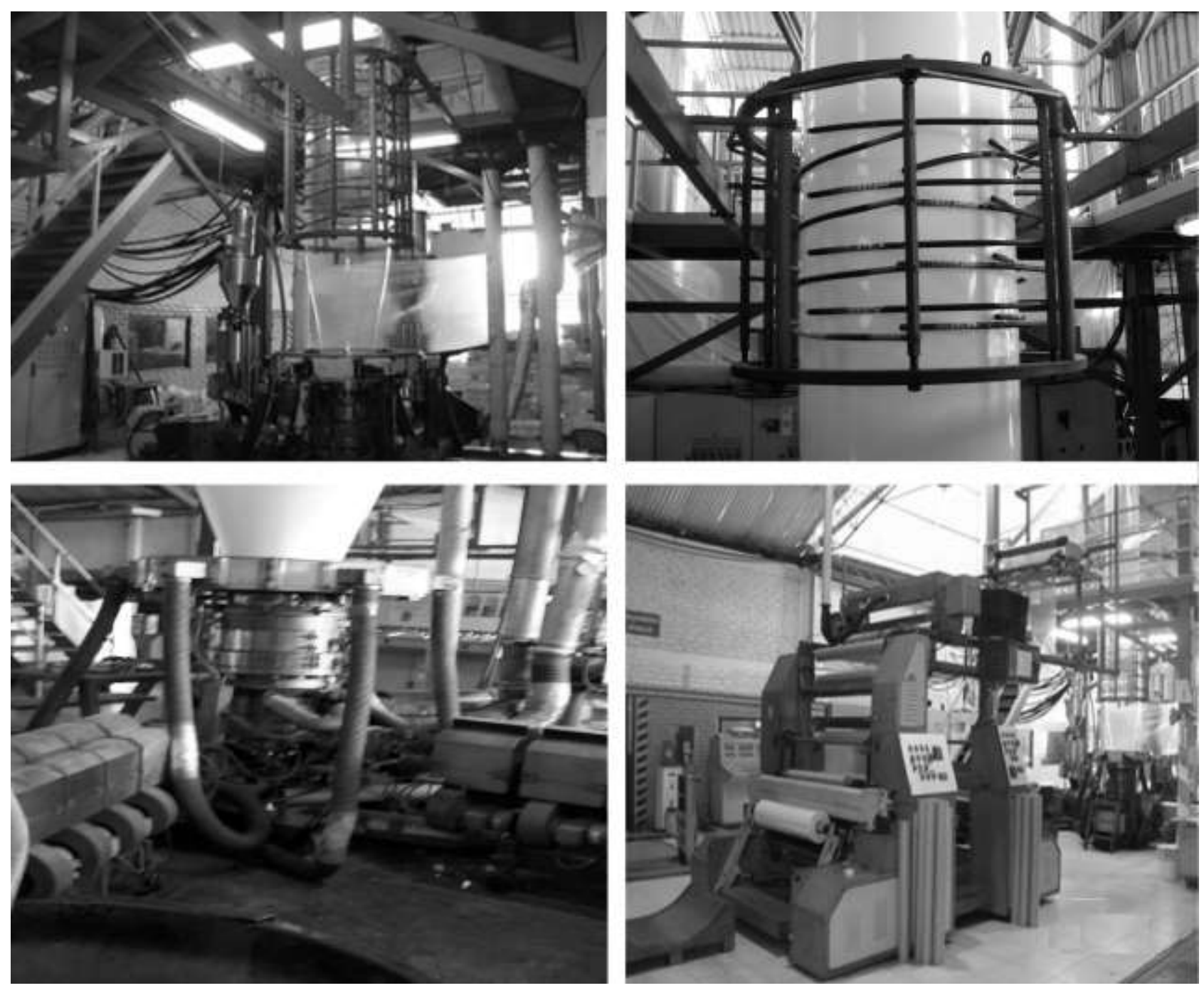

Fig. 3: Fotografías de la extrusora Ghioldi 1130

Tabla 10: Subsistema Coextrusor modelo 40A, 40B y 50C

\begin{tabular}{|c|c|c|c|c|c|c|c|}
\hline \multicolumn{8}{|c|}{ Clase: Co-Extrusor modelo $40 \mathrm{~A}, 40 B$ y $50 \mathrm{C}$} \\
\hline Sub Unit: & & & & & & & \\
\hline $\begin{array}{c}\text { Ítem } \\
\text { Mantenible }\end{array}$ & Motor principal & 150s3f & Transmisión & $\begin{array}{l}\text { uaja } \\
\text { reductora }\end{array}$ & $\begin{array}{l}\text { Iornillo } \\
\text { extrusor }\end{array}$ & Filtro & Cuello \\
\hline 1 & Taquimétrico & Resistencia & Polea & Engranaje & Tornillo sinfín & Portamalla & Resistencia \\
\hline 2 & $\begin{array}{l}\text { Controlador } \\
\text { motor }\end{array}$ & $\begin{array}{c}\text { Anillo de } \\
\text { calibración }\end{array}$ & Correa & Rodamiento & Camisa husillo & Malla & Pirómetro \\
\hline 3 & Rodamiento & Pirómetro & $\begin{array}{l}\text { Acoplamiento } \\
\text { motor }\end{array}$ & Lubricante & Resistencia & Resistencia & $\begin{array}{c}\text { Dispositivo } \\
\text { de actuación }\end{array}$ \\
\hline 4 & Bobinado & Termocupla & Lubricante & $\begin{array}{l}\text { Bomba de } \\
\text { aceite }\end{array}$ & Termocupla & Control & Termocupla \\
\hline 5 & $\begin{array}{c}\text { Dispositivo de } \\
\text { actuación }\end{array}$ & $\begin{array}{l}\text { Sensor de } \\
\text { presión }\end{array}$ & Sello & Filtro & Control & Termocupla & Control \\
\hline 6 & Monitorización & Control & $\begin{array}{c}\text { Acoplamiento } \\
\text { caja }\end{array}$ & Manguera & $\begin{array}{l}\text { Dispositivo de } \\
\text { actuación }\end{array}$ & $\begin{array}{l}\text { Sensor de } \\
\text { presión }\end{array}$ & \\
\hline 7 & Control & $\begin{array}{c}\text { Dispositivo } \\
\text { de actuación }\end{array}$ & & Llave & Pirómetro & & \\
\hline 8 & & Pinola & & $\begin{array}{l}\text { Sensor de } \\
\text { presión }\end{array}$ & $\begin{array}{c}\text { Turbina de } \\
\text { enfriamiento }\end{array}$ & & \\
\hline 9 & & $\begin{array}{l}\text { Tornillo de } \\
\text { calibración }\end{array}$ & & & & & \\
\hline
\end{tabular}

Detallado el equipo, se define la función del mismo y de los subsistemas. La función de la coextrusora 1130 
es: transformar un material sólido, usualmente granulado, por medio de la acción del calor y el trabajo mecánico, con temperaturas entre 120 y 220 grados centígrados por zona, en un material fundido que adquiera la forma deseada, al pasar por un orificio con una sección transversal predeterminada, para luego estirar y rotar el material, a una velocidad regulada, controlando el calibre de la película, y obteniendo una película con un calibre según formato de salida dado en micras, distribuyendo uniformemente cualquier variación en el espesor del material, evitando que se concentre en un solo punto de la bobina. El producto a obtener es una película con condiciones específicas como deslizamiento, brillo, y color (pigmento), con un tratamiento superficial realizado a través del electrodo de descarga con un voltaje de $10 \mathrm{kV} \mathrm{y} 20 \mathrm{kHz}$ aumentando la energía superficial de la película entre 37 y 40 dinas/cm, para terminar en bobinas de aproximadamente $300 \mathrm{~kg}$, con un ancho de $180 \mathrm{~cm}$ y un calibre dependiente de las necesidades del cliente.

La construcción del catálogo de fallas de la extrusora Ghioldi 1130, siguió la metodología indicada en la tabla 7, identificando 8 subsistemas, 18 fallas funcionales, 35 modos de falla, 174 causas de falla, 5 consecuencias de falla, y 174 actividades de mantenimiento recomendadas. La tabla 11 muestra parte del AMEF del subsistema Tratador electrónico de la extrusora Ghioldi 1130; el AMEF de los demás subsistemas y equipos siguió el mismo formato. Terminado el AMEF se sistematizan los catálogos de fallas en el software elaborado.

\section{Interfaz gráfica del sistema}

La interfaz de la herramienta, se implementó en macros de Excel, codificados en Visual Basic, e integró en un solo entorno, el ingreso de los datos y los resultados de la evaluación de la confiabilidad. El menú principal de la interfaz incorpora las opciones de catálogo de fallas y análisis de confiabilidad. En la figura 4 se presenta el registro de una falla en la interfaz de la herramienta desarrollada. El proceso de registro se realiza a través de una lista de opciones que se actualizan dependiendo de la elección en la opción anterior y la información del AMEF del equipo seleccionado, reduciendo los errores en la digitación de la información asociada a la falla. El registro es codificado, ordenado y almacenado en una hoja de cálculo, para su posterior análisis.

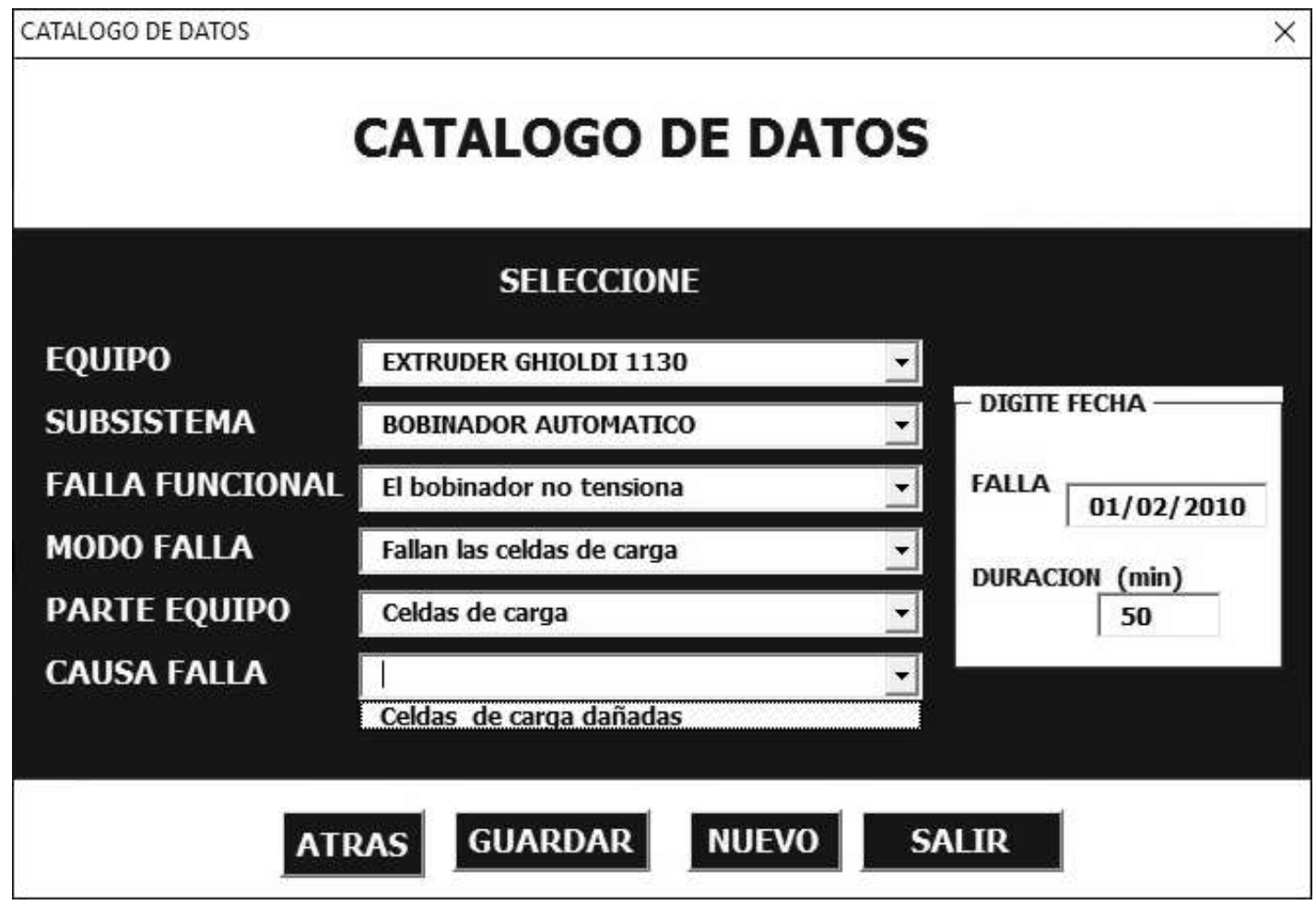

Fig.4: Catálogo de fallas de la herramienta

La opción análisis de confiabilidad, del menú principal, despliega una interfaz que permite al usuario seleccionar el equipo o subsistema del equipo, al que se requiere analizar la confiabilidad. Después de elegir, se despliega la interfaz de resultados que se muestra en la figura 5, y se genera un reporte como se aprecia en la tabla 12. La interfaz de resultados, presenta en la parte superior, la distribución que ajustó los datos de los registros de fallas; en la parte intermedia, los datos significativos de la función de confiabilidad $\mathrm{R}(\mathrm{t})$ y el MTBF; y en la parte inferior, la gráfica de la función de confiabilidad. 


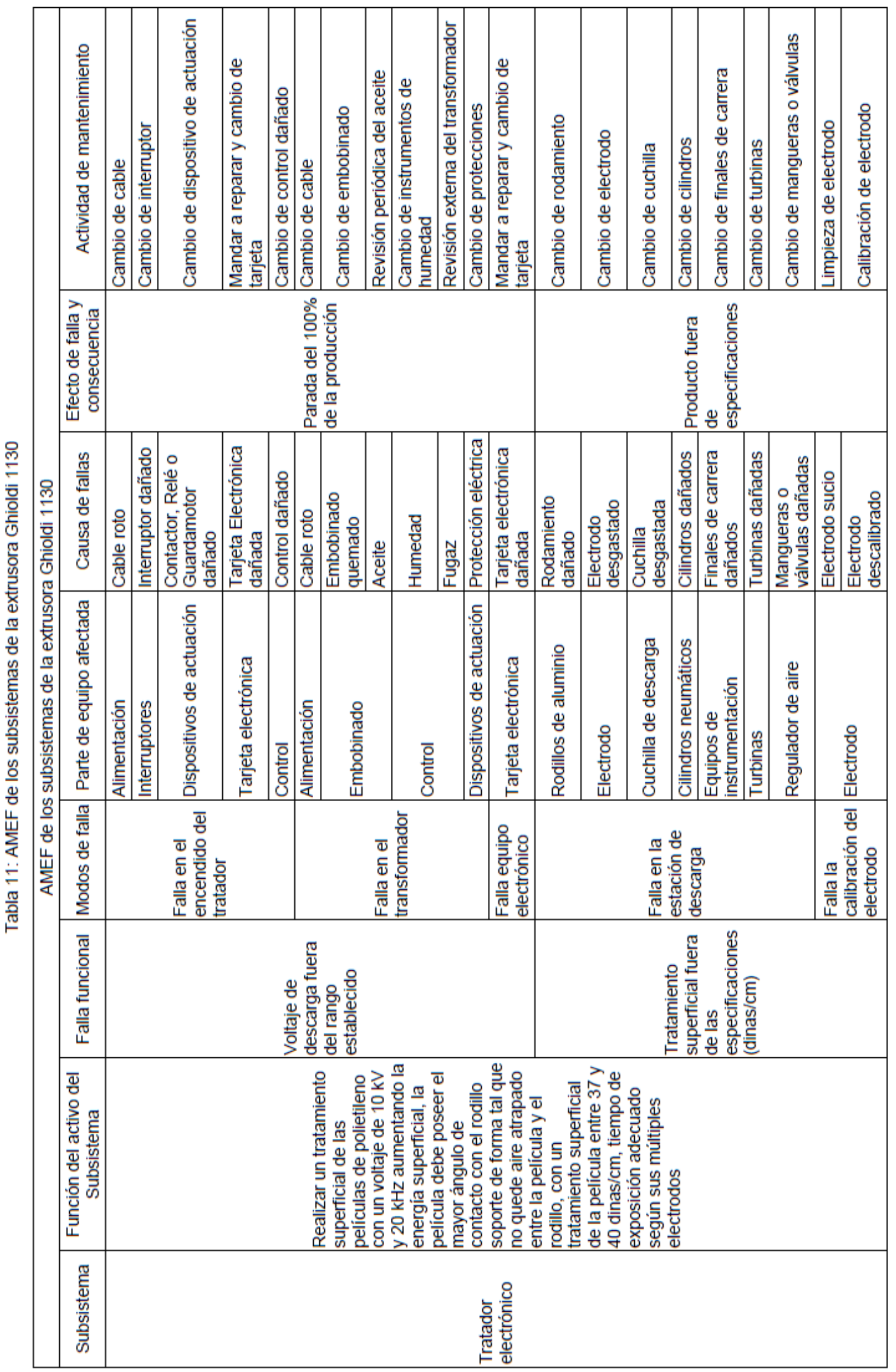




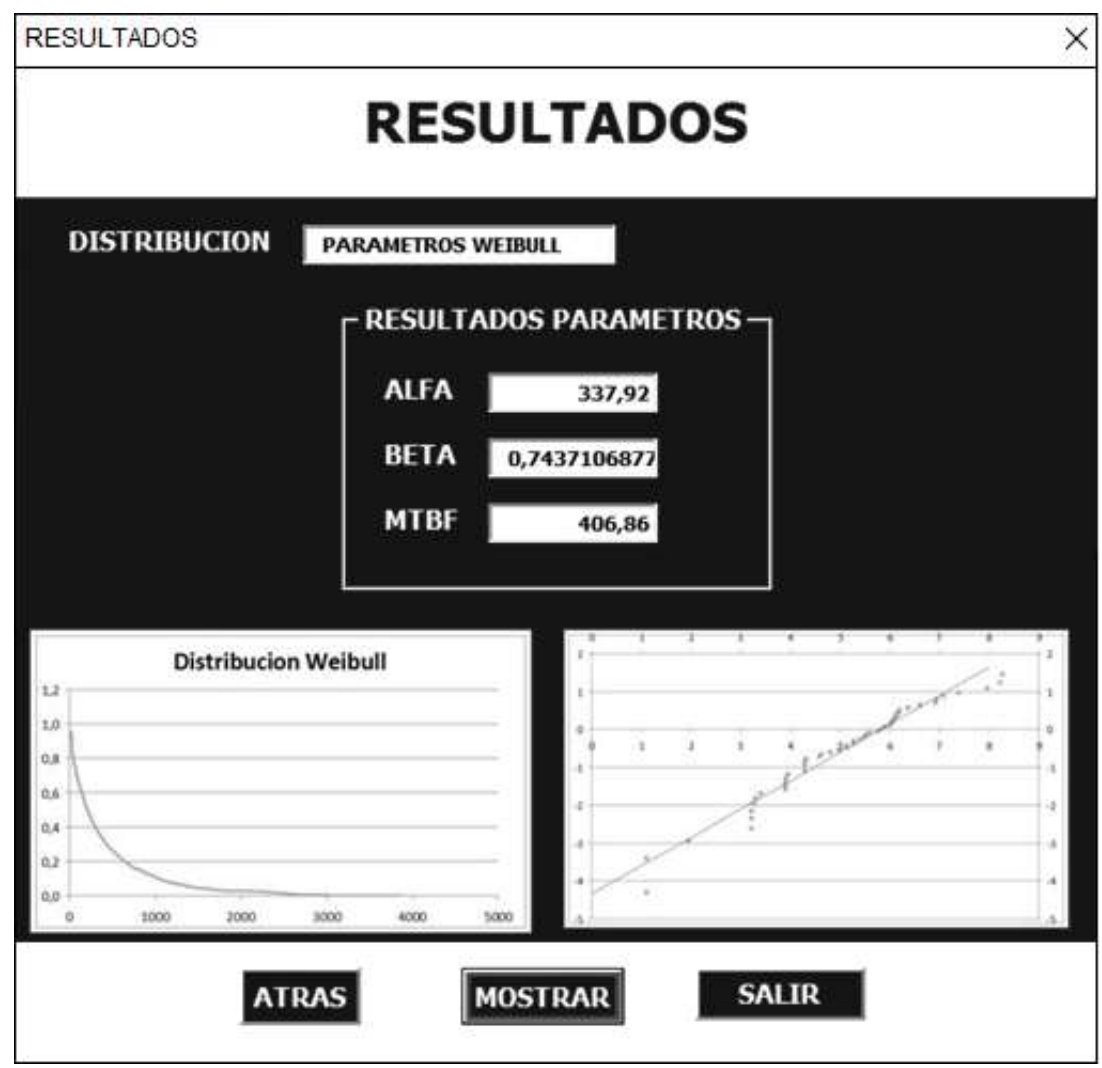

Fig. 5: Interfaz de resultados

Tabla 12: Resultados extrusora Ghioldi 1130

\begin{tabular}{|c|c|}
\hline Parámetro & Resultado \\
\hline Distribución & Weibull \\
\hline Kolmogorov-Smirnov, K-S D: & 0,0755 \\
\hline Cantidad de datos & 51 \\
\hline Pendiente de la recta $b$ & 0,7437 \\
\hline Intercepto de la recta $a$ & $-4,3304$ \\
\hline Coeficiente de correlación $r$ & 0,98164 \\
\hline Coeficiente de determinación muestral $r^{2}$ & 0,96363 \\
\hline Parámetro $\alpha$ de Weibull & 337,925 \\
\hline Parámetro $\beta$ de Weibull & 0,74371 \\
\hline Gamma $\boldsymbol{\Gamma}$ & 1,204 \\
\hline MTBF & 406,8616 \\
\hline
\end{tabular}

\section{Evaluación de la confiabilidad}

En la tabla 12, se muestra el reporte del análisis de la extrusora Ghioldi 1130. Los valores del coeficiente de correlación $r(0,98164)$ y el coeficiente de determinación muestral $r^{2}(0,96363)$, representan el ajuste de la recta de regresión, indicando la relación lineal entre los datos y el porcentaje de los puntos que están relacionados linealmente. La prueba de bondad de ajuste Kolmogorov-Smirnov, generó un $D$ de 0,0755, por lo tanto se aprueba la hipótesis de que los datos siguen la distribución de Weibull.

En la figura 5, se presentan los resultados del análisis de confiabilidad, de la extrusora Ghioldi 1130, en la interfaz gráfica. En esta figura se muestra la distribución de Weibull como la elegida, un MTBF igual a 406,8 horas, los parámetros alfa igual a 337,92 y beta igual a 0,74; y en la parte inferior la gráfica de $\mathrm{R}(\mathrm{t})$, que le permite al encargado del mantenimiento, responder a la pregunta: ¿cuál es la probabilidad de que la extrusora dure thoras sin fallar? Se puede observar que la confiabilidad de la extrusora 1130 a las 250 horas de operación, es del 0,45 y que después de 750 horas, tiene una probabilidad del $83 \%$ de fallar. 


\section{DISCUSIÓN FINAL}

A las empresas del sector de plástico que implementen el sistema para evaluar la confiabilidad, se les recomienda también generar catálogos de fallas a los equipos de mediana criticidad, para estandarizar el reporte de la mayoría de las fallas y disponer de información confiable sobre los principales equipos de la planta. También se recomienda, la capacitación del uso de la herramienta entre los trabajadores de las áreas de producción y mantenimiento, de tal forma que todos la conozcan y puedan utilizarla.

La confiabilidad de la planta, puede ser estimada utilizando la herramienta propuesta, a partir de las evaluaciones individuales de confiabilidad de los equipos y de la distribución de éstos en el proceso. La herramienta desarrollada está limitada a evaluar la confiabilidad, a partir de las distribuciones de Weibull, Log Normal y Normal, descartando en el análisis distribuciones como Poisson y Exponencial, que son ampliamente utilizadas en el periodo de vida normal de los equipos.

La implementación de la herramienta en Excel, facilitó la manipulación estadística de los registros y la elaboración de una interfaz gráfica. Esta interfaz aumentó la usabilidad del sistema porque integra en un mismo entorno, la entrada y salida de los datos.

\section{CONCLUSIONES}

De acuerdo con el estudio y los resultados presentados y discutidos, se obtienen las siguientes conclusiones:

Se puede inferir que en una empresa transformadora de plástico, el equipo de mayor criticidad es la extrusora, porque tiene un tiempo de operación prolongado y, costos y tiempos de mantenimiento altos. Además, una parada de este equipo, afecta el $100 \%$ de la producción. Estos indicadores impactan considerablemente en la competitividad de la industria; por tal motivo, se deben enfocar los esfuerzos y recursos del mantenimiento hacia este equipo, con el objetivo de mejorar su confiabilidad operacional.

La elaboración del catálogo de fallas a partir del AMEF, siguiendo los criterios de la norma SAE JA1011, SAE JA1012 y la ISO 14224, estandarizó los reportes de las averías en el sistema, garantizando que todas las fallas se describieran en el mismo formato y con los elementos necesarios para el análisis estadístico. Adicionalmente, la sistematización del catálogo de fallas, en la interfaz gráfica desarrollada para Excel, generó historiales de fallas de los equipos y subsistemas de forma efectiva y práctica, para la unidad de estudio.

EI MTBF calculado de forma dinámica y automática, a partir del historial de fallas, y no de la distribución uniforme de la vida útil dada por el fabricante, ofrece indicadores para la optimización de las actividades de mantenimiento preventivo. El MTBF del equipo crítico (Extrusora Ghioldi 1130), fue de 406,8 horas la primera vez que se ejecutó la aplicación, lo que implica diseñar actividades de mantenimiento con paradas planificadas antes de este tiempo, con el propósito de mejorar la confiabilidad del equipo.

\section{REFERENCIAS}

Altuger, G., Chassapis, C., Multi criteria preventive maintenance scheduling through Arena based simulation modeling, doi: 10.1109/WSC.2009.5429668, 1(1), 2123-2134 (2009)

Altmann, C., ¿Cómo mejorar la confiabilidad de un sistema complejo?, Revista Protección y Seguridad, 55 (325), 59-68 (2009)

Arata, A., Ingeniería y gestión de la confiabilidad operacional en plantas industriales, 1ª edición, 442-440, RiL Editores, Santiago de Chile (2009)

Bazovsky, I., Reliability theory and practice, 1aㅡ edición, 11-121, Dover Publications, New York, U.S.A. (2004)

Camps, L., Llanes, O., Silva, A., Fraga, H., An approach to fault diagnosis using meta-heuristics: a new variant of the differential evolution algorithm, doi: 10.13053/CyS-18-1-2014-015, Computación y Sistemas, 18 (1), 517 (2014)

Castillo-Serpa, A.M., Brito-Ballina, M.L., Fraga-Guerra,E., Análisis de criticidad personalizados, https://goo.gl/SVkhsK, ISSN: 1815-5944, Ingeniería Mecánica, 12 (3), 1-12 (2009)

Chemweno, P., Pintelon, L., Van Horenbeek, A., Muchiri, P., Development of a risk assessment selection methodology for asset maintenance decision making: An analytic network process (ANP) approach, doi: doi.org/10.1016/j.ijpe.2015.03.017, International Journal of Production Economics, 170 (B), $663-676$ (2015)

Cruse, T., Reliability-based mechanical design, 1a edición, 197-232, Marcel Dekker, New York, U.S.A. (1997) 
Duffuaa, S., Raouf, A., Dixon, J., Sistemas de mantenimiento: planeación y control, 1르 edición, 68-71, Limusa Wiley, México D.F., México (2009)

Espinosa, F. F., Días, A., Salinas, G. E., Un procedimiento para evaluar el riesgo de la innovación en la gestión del mantenimiento industrial, doi: 10.4067/S0718-33052012000200011, Ingeniare, Revista chilena de Ingeniería, 20(2), 242-254 (2012)

Espinosa, F.F., Días, A., Back, N., Un procedimiento de evaluación de las condiciones necesarias para innovar la gestión de mantenimiento en una empresa, doi: 10.4067/S0718-07642008000100012, Inf. Tecnol., 19(1), 97-104 (2008)

Flores, M.P., Torres, J.G., Rodríguez, J.H., Alcaraz, A.M., Confiabilidad Operativa de Sistemas para Compresión de Gas y Generación Eléctrica en Complejos Petroleros, doi: 10.1612/inf.tecnol.4366aybit.09, Inf. Tecnol., 21(3), 13-25 (2010)

Haldar, A., Mahadevan, S., Probability, reliability, and statistical methods in engineering design, $1^{\underline{a}}$ edición, 55-130, John Wiley, New York, U.S.A. (2000)

Holmberg, K., Folkeson, A., Bergman, B., Ostvik, R. Aage, C., Operational reliability and systematic maintenance, $1^{\underline{a}}$ edición, 247-324, Elsevier Science Publishers LTD, New York, U.S.A. (1991)

ISO 14224:2016, Petroleum, petrochemical and natural gas industries - Collection and exchange of reliability and maintenance data for equipment, 1-272, Ginebra, Suiza (2016)

Jiang, R., Zhang, K., Ma, Z., Wang, D., Fault mode, effects and criticality analysis for overheating fault of wind turbines gearbox and generator, doi: 10.1049/cp.2015.0445, International Conference on Renewable Power Generation (RPG 2015), 4(1), 1-5 (2015)

Mora, L., Mantenimiento. Planeación, ejecución y control, 1ª edición, 95-113, Alfaomega Grupo Editor, México D.F., México (2009)

Pintelon L. M., Gelders L.F., Maintenance management decision making, doi: 10.1016/0377-2217(92)90062E, European Journal of Operational Research, 58(3), 301-317 (1992)

Puthillath, B., Sasikumar, R., Selection of maintenance strategy using failure mode effect and criticality analysis, https://goo.gl/9feLRq, ISSN: 2277-3754, International Journal of Engineering and Innovative Technology (IJEIT), 1(6), 73-79 (2012)

SAE JA1011_200908, Evaluation criteria for reliability-centered maintenance (RCM) processes, 1-12, Washington, U.S.A. (2009)

SAE JA1012_201108, A guide to the reliability-centered maintenance (RCM) standard, 1-57, Washington, U.S.A. (2002)

Viveros P., Stegmaier R., Kristjanpoller F., Barbera L., Crespo A., Propuesta de un modelo de gestión de mantenimiento y sus principales herramientas de apoyo, doi: 10.4067/S0718-33052013000100011 Ingeniare. Revista chilena de Ingeniería, 21(1), 125-138 (2013)

Yañez, M., Gómez de la Vega, H., Valbueba, G., Ingeniería de confiabilidad y análisis probabilístico de riesgo, 1ae edición, 185-323, Reliability and Risk Management, México D.F., México (2004)

Yañez, M., Perdomo, J., Gómez de la Vega, H., Ingeniería de confiabilidad; Pilar fundamental del mantenimiento, https://goo.gl/YqKoNw. Acceso: 20 de enero (2016), Reliability and Risk Management, Venezuela (2002)

Zio, E., Reliability engineering: Old problems and new challenges, doi: 10.1016/j.ress.2008.06.002, Reliability Engineering and System Safety, 94(2), 125-141 (2009)

Sutrisno, A., Gunawan, I., Vanany, I., Khorshidhi, H. A., A maintenance waste risk appraisal model based on modified failure mode and effect analysis (FMEA), doi: 10.1109/IEEM.2016.7798112, 2016 IEEE International Conference on Industrial Engineering and Engineering Management (IEEM), 10(1), 1422-1425 (2016)

Valdes, M. E., Adapting failure mode and effects analysis (FMEA) to select hazard mitigation measures, doi: 10.1109/PCICON.2012.6549659, 2012 Petroleum and Chemical Industry Conference (PCIC), 59(1), 1-10 (2012) 